\title{
ADAPTIVE SUPPORT FOR POWER UNITS OF MACHINE-TRACTOR UNIT
}

Nikolay Egorov ${ }^{1}$, Farit Khaliullin ${ }^{1,2}$, Zulfia Khaliullina ${ }^{1}$, Larisa Zimina ${ }^{2}$

${ }^{1}$ Kazan State Agrarian University, Russia; ${ }^{2}$ Kazan National Research Technical University named after A. N. Tupolev, Russia

egnicholas333@gmail.com,khaliullin_kai_adis@mail.ru, khaliullinaz@mail.ru, larek.adis@mail.ru

\begin{abstract}
Under operating conditions, the power plant of a machine-tractor unit experiences changes in a wide range of changes in speed and load conditions. The vibrations arising from this, in addition to discomfort and additional loads on the components and mechanisms of the power plant, also cause excessive fuel consumption and power loss. Reducing the negative impact of vibration on effective performance of a power plant is proposed by creating supports with adjustable characteristics. The stiffness and damping coefficients were selected as the determined parameters. For tractor power plants, taking into account their layouts and design features, a design scheme with two attachment points in front with vertical movement and one hinge point at the rear was selected. As disturbing moments, we consider both the internal imbalance of the power plant itself and the external effects of the variable resistance moment and the shock of the supports themselves. Changes in the stiffness and damping coefficients of the supports used make it possible to reduce the oscillation amplitudes of the power plant under variable operating conditions, which will improve its utilization rates.
\end{abstract}

Keywords: power plant supports, effective indicators, stiffness, damping coefficient.

\section{Introduction}

The work of power plants of machine-tractor units is accompanied by a change in speed and load modes. The sources of such changes are both external and internal factors [1; 2]. Internal factors include the technological and structural imbalance of the engine and various technical conditions of its cylinders, and external factors include variable operating conditions of machine-tractor units [3]. All this leads to the appearance of vibration and vibrations of the engine framework, which, in turn, leads to a decrease in efficiency, to loss of power and to the appearance of additional variable loads on parts and assemblies, which ultimately reduces their life [4]. To combat this phenomenon, the parameters of the supports of power plants are selected with the possibility of neutralizing them.

\section{Status of the issue}

Currently, the parameters of the supports of power plants of machine-tractor units according to the recommendations are selected from the conditions:

1. the frequency range of disturbing factors is determined in accordance with the value of the reference frequency and the cutoff frequency after their harmonic analysis;

2. the stiffness of the support is selected according to the amplitude-frequency characteristics of the power unit and the value of its allowable value;

3. the damping characteristics of the supports are determined by the attenuation decrement of the power unit vibrations at principal frequencies of disturbing factors;

4. the selected parameters of the supports of the power unit are affected by its mass and dimensions and the installation diagram of these supports.

However, the proposed methodology for designing supports does not take into account the variability of the parameters of disturbing factors of both external and internal origin, which leads to a decrease in their operational efficiency outside of a fairly narrow, selected range [5]. Therefore, there is a need to create supports for power plants with adaptive characteristics [6].

\section{Proposed solution}

Consider the design scheme of the tractor (Figure 1), where, to simplify the calculations, an accepted is assumption on the equivalence of the supports of the left and right mounts, which makes it possible to switch to a flat design scheme. 


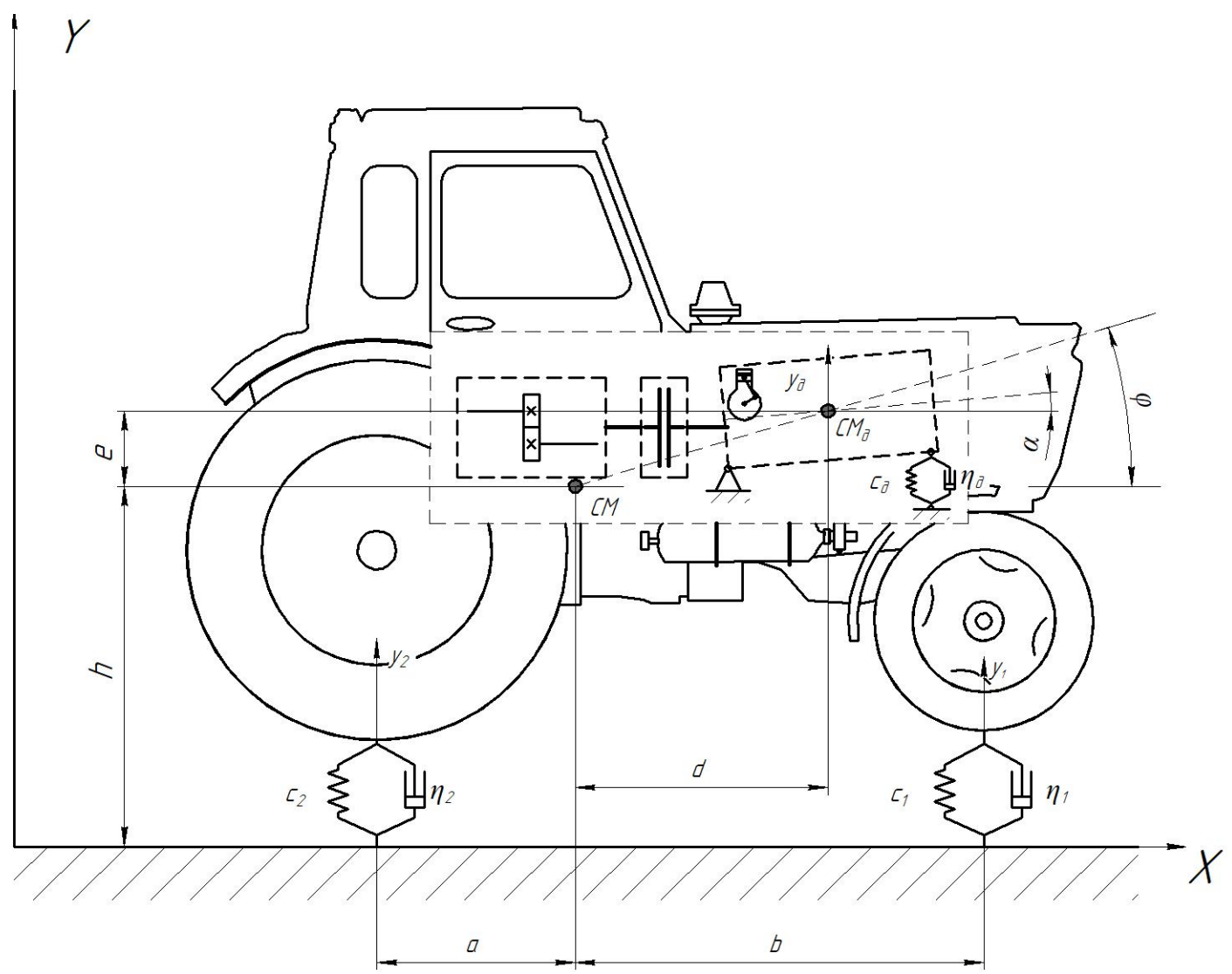

Fig. 1. Estimated scheme

Description of the generalized coordinates in Fig. 1 is as following:

- $y_{1}-$ displacement of tractor mass to the front axle;

- $y_{2}$ - displacement tractor mass to the rear axle;

- $\varphi$ - rotation angle of the framework relative to the center of mass;

- $y_{\partial}-$ displacement of the center of mass of the power unit.

Designations (Fig. 1):

- $m_{m p}, m_{\partial \varepsilon}, m_{1}, m_{2}$ - mass of the tractor and engine, the masses falling on the front and rear axles of the tractor, respectively;

- $\alpha_{1}, \alpha_{2}, \alpha_{\partial}$-damping coefficients of the front axle supports, rear axle, power unit supports, respectively;

- $c_{1}, c_{2}, c_{\partial}$-stiffness of the supports of the front axle, rear axle, supports of the power unit, respectively;

- $J_{0}$ - moment of inertia of the framework relative to the center of mass;

- $d$-distance from the axis of the center of mass to the center of mass of the engine;

- $h$ - distance from the supporting surface to the center of mass of the tractor.

The design scheme of the tractor is its simplified view, in front of which there is a power unit, presented as a hard body, mounted on an absolutely rigid base with two supports, the rear of which is an articulated connection of the power unit with the base, and the front one is an elastic-damping support with the possibility of linear movements. Such a scheme maximally reflects the design features of placing a power plant on the frame of a tractor and, at the same time, allows a more detailed assessment of the vibrational (vibrational) loads excited by the engine and transmitted through the supports of the power unit.

If we consider the framework of the tractor as a solid body, capable of shifting in any direction and thus having six degrees of freedom, then to determine its position in space, six generalized coordinates must be specified. As such coordinates, three Cartesian coordinates of the center of mass of the framework and three angles that specify the rotations of the coordinate axes, rigidly connected with it, relative to the fixed coordinate axes, are usually taken. The selected estimated scheme is 
presented in the longitudinal section (Figure 1), which allows to limit moving in the vertical plane the front $-y_{1}$ and rear axles $-y_{2}$, and the rotation angle of the framework relative to the center of mass $\varphi$, vibrations of the power plant are expressed through vertical displacements of its center of mass $-y_{\partial}$.

To compile the mathematical model, some simplifications were accepted, which, with a slight decrease in the accuracy of the calculations, leave an adequate picture of the vibration of the object under study [7-9].

When modeling, the following general restrictions were adopted:

- the power unit is an absolutely rigid body;

- the frame (framework) also represents an absolutely rigid body;

- power unit - concentrated mass;

- torsional vibrations arising in the transmission of the machine are not considered;

- oscillations of the power unit caused by constantly acting disturbances occur in the direction of action of these disturbances. Then the oscillations of the power unit in various directions are not connected, and they can be calculated separately, considering in each case the power unit as the simplest oscillatory system with one degree of freedom;

- the elastic deformations of the power unit caused by the deformation of the parts, the rigidity of the joints between the parts, because of what is possible the displacement of the individual parts making up the power unit are taken into account;

- the elastic characteristics of the supports of the power unit - are linear. In reality, the rubbermetal supports of the power unit have non-linear elastic characteristics.

To describe the oscillatory motion, we use the second-order Lagrange equations, which after all transformations have the following form:

- Lagrange equation with respect to $y_{1}$ for the design scheme

$$
m_{1} \ddot{y}_{1}+\alpha_{1} \dot{y}_{1}+c_{1} y_{1}-\alpha_{1} \dot{y}-c_{1} y-\alpha_{1} b \dot{\varphi}-c_{1} b \dot{\varphi}=F_{1}(t),
$$

- Lagrange equation with respect $y_{2}$ for the design scheme

$$
\begin{gathered}
m_{2} \ddot{y}_{2}+\left(\alpha_{2}+\alpha_{\partial} \frac{d^{2}}{a^{2}}\right) \dot{y}_{2}+\left(c_{2}+c_{\partial} \frac{d^{2}}{a^{2}}\right) y_{2}+ \\
+\left(\alpha_{2} a-\alpha_{\partial} \frac{d^{2}}{a}\right) \dot{\varphi}+\left(c_{2} a-c_{\partial} \frac{d^{2}}{a}\right) \varphi-\alpha_{\partial} \frac{d}{a} \dot{y}_{\partial}-c_{\partial} \frac{d}{a} y_{\partial}=F_{2}(t),
\end{gathered}
$$

- Lagrange equation with respect $\varphi$ for the design scheme

$$
\begin{aligned}
& J_{0} \ddot{\varphi}+\left(\alpha_{1} b^{2}+\alpha_{2} a^{2}+\alpha_{\partial} d^{2}\right) \dot{\varphi}+\left(c_{1} b^{2}+c_{2} a^{2}+c_{\partial} d^{2}\right) \varphi-\alpha_{1} b \dot{y}_{1}-c_{1} b y_{1}+ \\
& +\left(\alpha_{2} d-\alpha_{\partial} \frac{d^{2}}{a}\right) \dot{y}_{2}+\left(c_{2} a-c_{\partial} \frac{d^{2}}{a}\right) y_{2}+\alpha_{\partial} d \dot{y}_{\partial}+c_{\partial} d y_{\partial}=F_{\varphi}(t) .
\end{aligned}
$$

We write down the Lagrange equation with respect to $y_{\partial}$ for the design scheme:

$$
m_{\partial} \ddot{y}_{\partial}+c_{\partial} \dot{y}_{\partial}+c_{\partial} y_{\partial}-\alpha_{\partial} \dot{y}_{\partial}-c_{\partial} y+\alpha_{\partial} d \dot{\varphi}+c_{\partial} d \varphi-\alpha_{\partial} \frac{d}{a} \dot{y}_{2}-c_{\partial} \frac{d}{a} y_{2}=F_{\partial}(t),
$$

where $F_{1}(t), F_{2}(t), F_{\varphi}(t), F_{\partial}(t)$ - generalized perturbing forces corresponding to the generalized coordinates $y_{1}, y_{2}, \varphi, y_{\partial}$.

The obtained system of equations allows us to determine the parameters of the arising oscillations for any form of disturbing force. The main analytical difficulties in solving the equations described above are associated with cumbersome transformations in the expressions of potential and kinetic energy and their subsequent differentiation by generalized coordinates and time. These difficulties were overcome through the use of computers and the software package [5] MathWorks MATLAB, designed to solve problems of mathematical and technical calculations. A great advantage of working with this software package is that based on a fairly simple description of the model, as close as 
possible to traditional calculation, the calculation is automatically performed and the result of systems of differential equations of oscillations is formed. Thus, the formed equations are solved by numerical methods [6].

For the numerical experiment according to the proposed mathematical model, an MTZ82 tractor with a D-243 engine was chosen, the parameters of which are given below:

- $\quad$ tractor weight $m_{m p}=3900 \mathrm{~kg}$;

- engine mass $m_{\partial \delta}=430 \mathrm{~kg}$;

- tractor base $2450 \mathrm{~mm}$;

- height of the center of gravity of the tractor $h=1200 \mathrm{~mm}$;

- distance from the axis of the center of mass to the center of mass of the engine $d=1000 \mathrm{~mm}$;

- $\quad$ stiffness of the support of the power unit $s d=300 \mathrm{kN} \cdot \mathrm{m}^{-1}$;

- damping coefficient of the power unit supports $\alpha d=3000 \mathrm{~N} \cdot\left(\mathrm{m} \cdot \mathrm{s}^{-1}\right)^{-1}$.

The missing design parameters were selected according to the recommendations of literary sources [10-13].

The option of using the supports of a power plant with various characteristics was considered [14]: stiffness of the power unit support $\mathrm{sd}=250 \mathrm{kN} \cdot \mathrm{m}^{-1}$ (

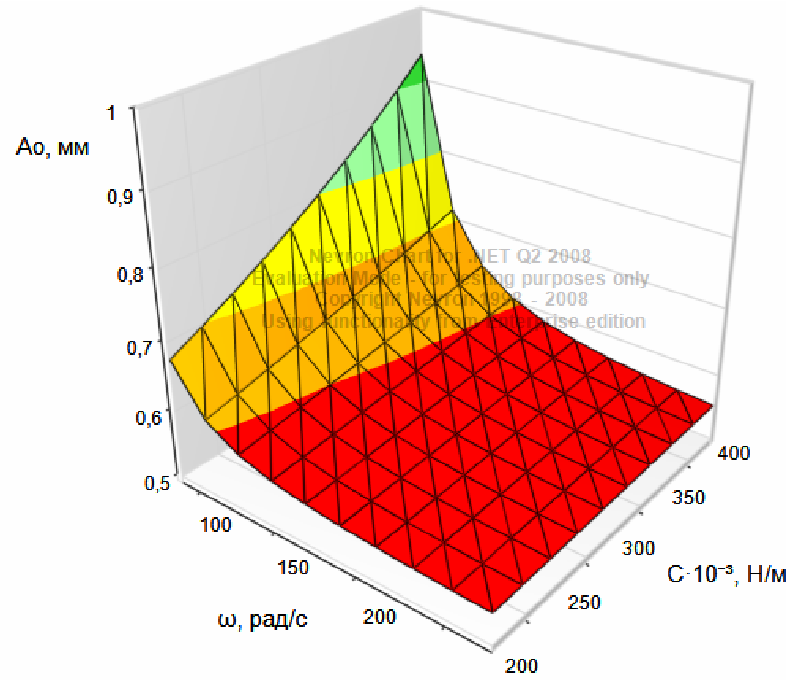

Fig. 2. Effect of the stiffness of the supports of the power unit on the amplitude at various speed conditions

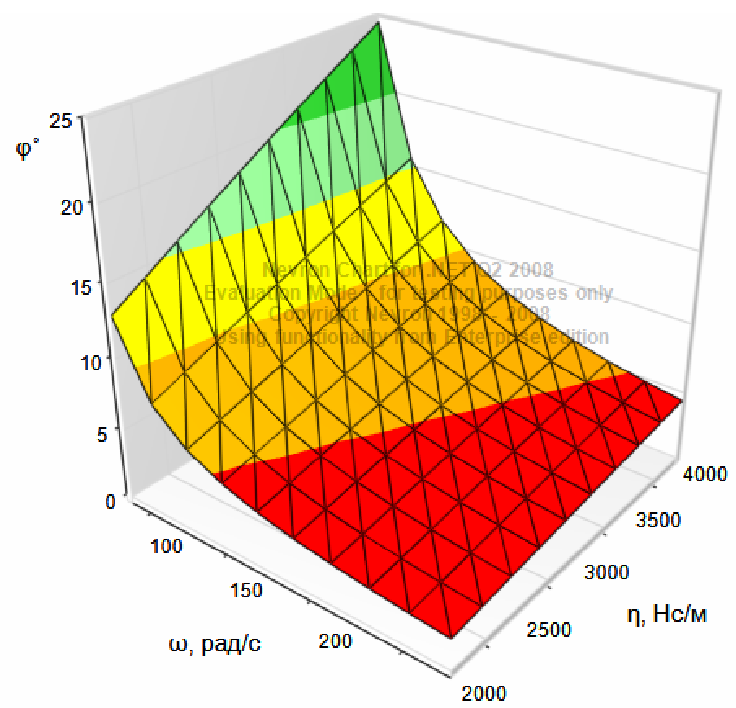

Fig. 3. Influence of the damping coefficient of the power unit supports on the amplitude at various speed conditions 


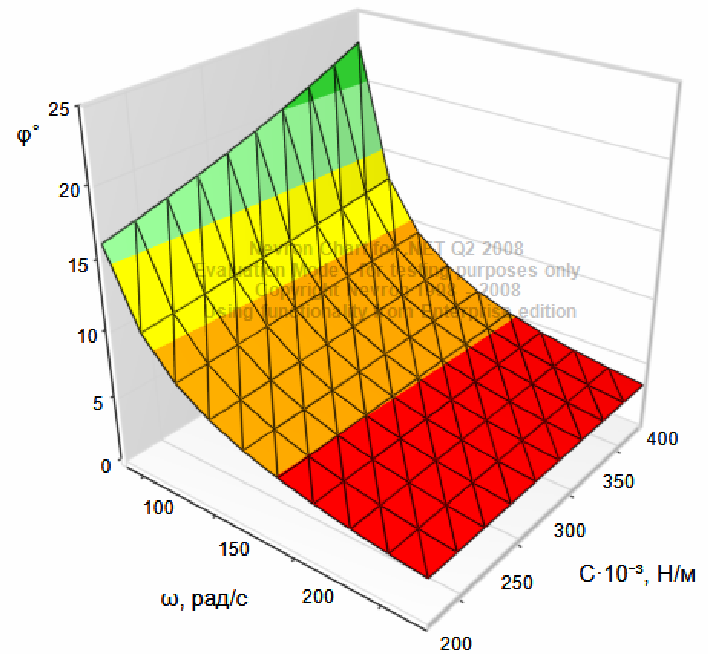

\section{Fig. 4. Effect of the stiffness of the supports of the power unit on the phase shift of the oscillations at various speed conditions}

The analysis of the numerical studies shows that the speed mode has a more significant influence on the amplitude of oscillations and on their phase shift than changes in the rigidity of the supports and their damping coefficient. So, when the frequency decreases from 200 to $50 \mathrm{rad} \cdot \mathrm{s}^{-1}$, the amplitude of the oscillations increases from $0.55 \mathrm{~mm}$ to $0.75 \mathrm{~mm}$, and with a decrease in stiffness, the intensity of this effect weakens (Fig. 2). The influence of the damping coefficient of the power unit supports on the oscillation amplitude is also more pronounced at low frequencies (Fig. 3). An increase in the rigidity of the supports of a power plant at a constant frequency increases the phase shift of the oscillation amplitude of the power unit (Fig. 4).

\section{Conclusions}

A mathematical model of the oscillatory motion of the power plant and the framework of a machine-tractor unit with an arbitrary disturbing factor at an algorithm for solving it is proposed, which allows us to determine the dependence of the parameters of this movement on the characteristics of the disturbing forces and moments. Adopted assumptions and simplifications of the design scheme allow us to switch to a flat oscillatory scheme, which allows us to significantly simplify the calculations at the preliminary stage of the research. The obtained results allow to propose an improved methodology for determining the indices of the supports of a power plant, which can more effectively reduce the amplitude of oscillations in a wide range of speed and load modes.

\section{References}

[1] Wect J.P. Hydraulically-damped engine-mounting// Automotive Engineer. - 1987 -V.12, No.1, pp. 17-19.

[2] Egorov N.M., Khalyullin F.K. Stand for test of support in structure of suspension of power unit// Materials of the I International scientific and practical conference dedicated to the memory of Professor A.K. Yuldashev: Dynamics of mechanical systems, 5-6 April 2018, Kazan.

[3] Dodtibacher D. Rechnerische Ermittlung des Schwingverhaltens des elastischge-lagerten Motors $\mathrm{imPkm} / /$ Automobil-Indastrie. -1982. -No. 1. pp. 57-61. (In German)

[4] Khaliullin F., Aladashvili J.K., Nurmiev A.A., Pikmullin G.V., Sinitsky S.A. Determination of statistical data of conditional probabilities of the technical condition of internal combustion engines when compiling the Bayes diagnostic table// IOP Conference Series: Materials Science and Engineering, Volume 635, 10th International Conference on Mechatronics and Manufacturing (ICMM 2019) 21-23 January 2019, Bangkok, Thailand, DOi: 10.1088/1757-899X/635/1/01/2017

[5] Flower W.S. Understanding Hudraulic Mounts for Improved Vehicle Noise, Vi-bration and Ride Qualitits// SAE paper, 1985. -No. 850975. pp. 123-132.

[6] Novokshenov V.K. Reducing low-frequency vibration of a power unit with a transverse engine: diss cand. tech. Sciences 05.05.03: - M., 1986.- 226 p. 
[7] Adomian J. Stochastic Systems: Trans. from English - M .: Mir, 1987.- 376 p.

[8] Construction of dynamic stochastic models from experimental data. Kashyap R.L., Rao A.R. Per from English. - M .: Science. The main edition of the physical and mathematical literature, 1983. $384 \mathrm{p}$.

[9] Zarubin V.S. Mathematical modeling in technology: textbook. for universities/V.S.Zarubin.- 3rd ed. - M.: Publishing House of MSTU. N.E.Bauman, 2010, - 495 p.

[10] Kutkov G.M. Tractors and cars. Theory and technological properties. - M .: KlosS, 2004. - 504 p.

[11]Tracts. Design: Textbook for university students/I.P. Ksenevich, V.M. Sharipov, L.Kh. Arustamov and others; Under the total. ed. I.P. Ksenevich, V.M. Sharipov. - M.: Mechanical Engineering, 2000. $821 \mathrm{p}$.

[12] Kaptyushin G.K., Bazhenov S.P. Design, theory, design and testing of tractors. - M.: Agropromizdat, 1990. $511 \mathrm{p}$.

[13] Tractors "Belarus" MTZ-80, MTZ-80L, MTZ-82, MTZ-82L: Tech. description and instruction manual/N.V. Matyukhov, I.F. Bruenkov, E.A. Bomberov, etc. - 2nd ed., revised. and add. - Mn .: Urajay, 1981. $352 \mathrm{p}$.

[14] Egorov N.M., Khalyullin F.K. Adaptive support of power units// Materials of the I International scientific and practical conference dedicated to the memory of Professor A.K. Yuldashev: Dynamics of mechanical systems, 5-6 April 2018, Kazan. 